\title{
Optimization of benzoxazinones as natural herbicide models by lipophilicity enhancement.
}

\section{Supporting information}

Francisco A. Macías, * David Marín, Alberto Oliveros-Bastidas, José M.G. Molinillo

Grupo de Alelopatía, Departamento de Química Orgánica, Universidad de Cádiz. C/ Avda. República Saharaui s/n, 11510-Puerto Real, Cádiz, Spain

*To whom correspondence should be addressed; Phone: +34-956.016.370; Fax: +34956.016.193; e-mail: famacias@uca.es. 


\section{Physical data}

Please note: Structures are numbered as in the article, with side chains starting from C11 (carboxyl group) to the end of the chain:

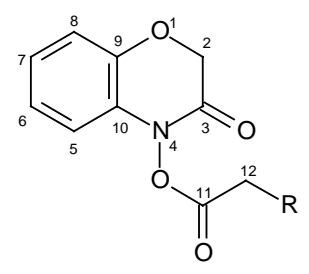

4-Hydroxy-(2H)-1,4-benzoxazin-3(4H)-one (D-DIBOA), 4-acetoxy-(2H)-1,4benzoxazin-3(4H)-one (ABOA): Their spectroscopic data matched exactly to those previously reported (see Atkinson, J.; Morand, P.; Arnason, J. T.; Niemeyer, H. M.; Bravo, H. R. Analogues of the cyclic hydroxamic acid 2,4-dihydroxy-7-methoxy-2H1,4-benzoxazin-3-one (DIMBOA): decomposition to benzoxazolinones and reaction with b-mercaptoethanol. J. Org. Chem. 1991, 56, 1788-1800).

4-Propanoyloxy-(2H)-1,4-benzoxazin-3(4H)-one (Pr-D-DIBOA): FTIR ( $\left.\mathbf{c m}^{-\mathbf{1}}\right)$ : 2924 , 2853, 1692, 1494, 1277, 1049, 752; ${ }^{1} \mathbf{H} \mathbf{~ N M R ~}\left(\mathbf{C D C l}_{3}, 400 \mathbf{M H z}\right) \delta 1.32[\mathrm{t}, 3 \mathrm{H}, J=8$ $\mathrm{Hz}(\mathrm{H}-12)], 2.69$ [m, 2H (H-11)], 4.76 [wd, 2H, J = $10 \mathrm{~Hz}(\mathrm{H}-2), 6.84$ [dd, 1H, J = 2, 9 $\mathrm{Hz}(\mathrm{H}-5)], 7.00[\mathrm{~m}, 3 \mathrm{H}(\mathrm{H}-6, \mathrm{H}-7, \mathrm{H}-8)] .{ }^{13} \mathbf{C} \mathbf{~ N M R}\left(\mathbf{C D C l}_{3}, \mathbf{1 0 0} \mathbf{M H z}\right) \delta 9.33[\mathrm{t}(\mathrm{C}-$ 13)], 24.9 [t (C-12)], 68.5 [t (C-2)], 112.2 [d (C-8)], 117.3 [d (C-6)], 123.2 [d (C-5)], 125.3 [d (C-7)], 128.4 [s (C-10)], 143.6 [s (C-9)], 159.3 [s (C-3)], 169.8 [s (C-11)]. EIMS (probe, $70 \mathrm{eV}) \mathrm{m} / \mathrm{z} 221.3[\mathrm{M}]^{+}(3 \%)$.

4-Butanoyloxy-(2H)-1,4-benzoxazin-3(4H)-one (Bu-D-DIBOA): FTIR (cm $\left.{ }^{-1}\right)$ : 2924, 2853, 1805, 1721, 1711, 1495, 1276, 1049, 750; ${ }^{\mathbf{1}} \mathbf{H} \mathbf{~ N M R}\left(\mathbf{C D C l}_{3}, 400 \mathbf{~ M H z}\right) \delta 1.07$ [t, $3 \mathrm{H}, \mathrm{J}=9 \mathrm{~Hz}(\mathrm{H}-14)], 1.82$ [ddq, 2H, J = 9, 9, $7 \mathrm{~Hz}(\mathrm{H}-13)], 2.63$ [dd, 2H, J = 7, $9 \mathrm{~Hz}$ (H-12)], 6.83 [m, 2H (H-5, H-6)], 7.00 [m, 2H (H-7, H-8)]. ${ }^{13} \mathbf{C}$ NMR $\left(\mathbf{C D C l}_{3}, \mathbf{1 0 0}\right.$ MHz) $\delta 13.8$ [q (C-14], 18.5 [t (C-13)], 33.2 [t (C-12)], 68.45 [t (C-2)], 112.4 [d (C-8)], 117.1 [d (C-6)], 123.0 [d (C-5)], 125.0 [d (C-7)], 128.2 [s (C-10)], 143.9 [s (C-9)], 159.4 [s (C-3)], 169.3 [s (C-11)]. EIMS (probe, $70 \mathrm{eV}) \mathrm{m} / \mathrm{z} 235.5[\mathrm{M}]^{+}(5 \%)$.

4-Valeroyloxy-(2H)-1,4-benzoxazin-3(4H)-one (Val-D-DIBOA): FTIR ( $\mathbf{c m}^{-\mathbf{1}}$ ): ${ }^{1} \mathbf{H}$ NMR $\left(\mathbf{C D C l}_{3}, 400 \mathrm{MHz}\right) \delta 0.95[\mathrm{t}, 3 \mathrm{H}, \mathrm{J}=7 \mathrm{~Hz}(\mathrm{H}-14)], 1.45$ [ddq, 2H, J = 7, 15, $\left.15(\mathrm{H}-13)\right]$, 1.76 [dddd, 2H, J = 7, 15, 7, $15(\mathrm{H}-13)], 2.63$ [dd, 2H, J = 7, $15(\mathrm{H}-12)], 4.74$ [d, 2H, J = $32(\mathrm{H}-2)], 26.82$ [m, 2H (H-5, H-6)], 6.98 [m, 2H (H-7, H-8)]. ${ }^{13} \mathbf{C}$ NMR $\left(\mathbf{C D C l}_{3}, \mathbf{1 0 0}\right.$ MHz) $\delta$ 13.5 [q (C-15)], 22.1 [t, (C-14)], 26.6 [t (C-13)], 30.8 [t (C-12)], 68.1 [t (C-2)], 112.1 [d (C-8)], 116.9 [d (C-6)], 122.7 [d (C-5)], 124.7 [d (C-7)], 127.9 [s (C-10)], 143.6 [s (C-9)], 159.13 [s (C-3)], 169.1 [s (C-11)]. EIMS (probe, 70 eV) m/z 249.2 [M] ${ }^{+}$ $(3 \%)$.

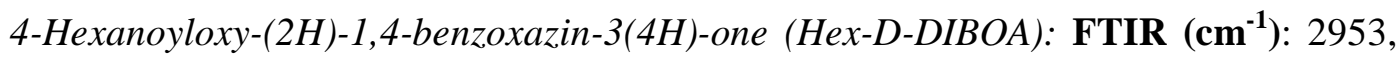
2928, 2862, 1812, 1719, 1692, 1489, 1278, 1043, 759; ${ }^{1} \mathbf{H}$ NMR $\left(\mathbf{C D C l}_{3}, 400 \mathbf{~ M H z}\right) \delta$ 0.91 [t, 3H, J = 7 (H-16)], 1.37 [m, 4H (H-15, H14)], 1.78 [dddd, 2H, J = 3, 7, 7, $15(\mathrm{H}-$ 
13)], 2.63 [dd, 2H, J = 7, 15 (H-12)], 4.76 [d, 2H, J = 9 (H-2)], 6.83 [ddd, 1H, J = 8, 8, 2 $(\mathrm{H}-6)], 7.00$ [m, 3H (H-5, H-7, H-8)]. ${ }^{13} \mathbf{C ~ N M R}\left(\mathbf{C D C l}_{3}\right.$, 100 MHz) $\delta 13.7$ [q (C-16)], 22.1 [t (C-15)], 24.3 [t (C-13)], 31.0 [t, 2C (C-12, C-14)], 68.1 [t (C-2)], 112.1 [d (C8)], 116.8 [d (C-6)], 122.7 [d (C-5)], 124.8 [d (C-7)], 127.8 [s (C-10)], 143.5 [s (C-9)], 159.1 [s (C-3)], 169.2 [s (C-11)]. EIMS (probe, $70 \mathrm{eV}) \mathrm{m} / \mathrm{z} 263.1[\mathrm{M}]^{+}(2 \%)$.

4-Heptanoyloxy-(2H)-1,4-benzoxazin-3(4H)-one (Hept-D-DIBOA): FTIR $\left(\mathbf{c m}^{-\mathbf{1}}\right)$ : 2956 , 2931, 2859, 1805, 1711, 1496, 1692, 1496, 1276, 1049, 751; ${ }^{\mathbf{H}} \mathbf{H M R}\left(\mathbf{C D C l}_{3}, \mathbf{4 0 0}\right.$ MHz) $\delta 0.89[\mathrm{t}, 3 \mathrm{H}, \mathrm{J}=7(\mathrm{H}-17)], 1.37$ [m, 6H (H-16, H-15, H-14)], 1.78 [dddd, 2H, J $=7,15,7,15(\mathrm{H}-13)], 2.64[\mathrm{dd}, 2 \mathrm{H}, \mathrm{J}=7,14(\mathrm{H}-12)], 4.75[\mathrm{~d}, 2 \mathrm{H}, \mathrm{J}=9(\mathrm{H}-2)], 6.83$ [ddd, 1H, J = 8, 8, 2 (H-6)], $7.00[\mathrm{~m}, 3 \mathrm{H}(\mathrm{H}-5, \mathrm{H}-7, \mathrm{H}-8)] .{ }^{13} \mathbf{C} \mathbf{~ N M R}\left(\mathbf{C D C l}_{3}, \mathbf{1 0 0}\right.$ MHz) $\delta 13.9$ [q (C-17)], 22.3 [t (C-16)], 24.5 [t (C-13)], 28.5 [t (C-14)], 31.1 [t, 2C (C12, C-15)], 68.1 [t (C-2)], 112.1 [d (C-8)], 116.8 [d (C-6)], 122.7 [d (C-5)], 124.8 [d (C7)], 127.8 [s (C-10)], 143.5 [s (C-9)], 159.1 [s (C-3)], 169.2 [s (C-11)]. EIMS (probe, $70 \mathrm{eV}) \mathrm{m} / \mathrm{z} 277.1[\mathrm{M}]^{+}(35 \%)$.

4-Octanoyloxy-(2H)-1,4-benzoxazin-3(4H)-one (Oct-D-DIBOA): FTIR (cm $\left.{ }^{-1}\right): 2955$, 2928, 2857, 1794, 1691, 1496, 1276, 1050, 750; ${ }^{1} \mathbf{H} \mathbf{~ N M R}\left(\mathbf{C D C l}_{3}, \mathbf{4 0 0} \mathbf{~ M H z}\right) \delta 0.80[\mathrm{t}$, $3 \mathrm{H}, \mathrm{J}=7$ (H-18)], 1.21 [m, 8H (H-17, H-16, H-15, H-14)], 1.70 [dddd, 2H, J = 7, 15, 5, 15 (H-13)], 2.56 [dd, 2H, J = 5 (H-12)], 4.66 [d, 2H, J = 7 (H-2)], 6.76 [ddd, 1H, J = 8, 8, 2 (H-6)], $6.90[\mathrm{~m}, 3 \mathrm{H}(\mathrm{H}-5, \mathrm{H}-7, \mathrm{H}-8)] .{ }^{13} \mathbf{C ~ N M R}\left(\mathbf{C D C l}_{3}, \mathbf{1 0 0} \mathbf{~ M H z}\right) \delta 13.8[\mathrm{q}(\mathrm{C}-$ 18)], 22.3 [t (C-17)], 24.5 [t (C-13)], 28.6 [t (C-14)], 28.7 [t (C-15)], 30.9 [t (C-12)], 31.3 [t (C-16), 67.9 [t (C-2)], 111.8 [d (C-8)], 116.5 [d (C-6)], 122.4 [d (C-5)], 124.4 [d (C-7)], 127.6 [s (C-10)], 143.3 [s (C-9)], 158.8 [s (C-3)], 168.8 [s (C-11)]. EIMS (probe, $70 \mathrm{eV}) \mathrm{m} / \mathrm{z} 291.4[\mathrm{M}]^{+}(2 \%)$.

4-Nonanoyloxy-(2H)-1,4-benzoxazin-3(4H)-one (Non-D-DIBOA): FTIR (cm $\left.{ }^{-1}\right)$ : 2955 , 2926, 2855, 1794, 1709, 1495, 1276, 1049, 748; ${ }^{\mathbf{1}} \mathbf{H} \mathbf{~ N M R}\left(\mathbf{C D C l}_{3}, 400 \mathbf{~ M H z}\right) \delta 0.82$ [t, 3H, J = 4 (H-19)], 1.22 [m, 10H (H-18, H-17, H-16, H-15, H-14)], 1.72 [dddd, 2H, J $=7,15,7,15(\mathrm{H}-13)], 2.58[\mathrm{dd}, 2 \mathrm{H}, \mathrm{J}=7,15(\mathrm{H}-12)], 4.69$ [d, 2H, J = $7(\mathrm{H}-2)], 6.78$ [ddd, $1 \mathrm{H}, \mathrm{J}=8,8,2$ (H-6)], 6.94 [m, 3H (H-5, H-7, H-8)]. ${ }^{13} \mathbf{C}$ NMR $\left(\mathbf{C D C l}_{3}, \mathbf{1 0 0}\right.$ MHz) $\delta 13.9$ [q (C-19)], 22.4 [t (C-18)], 24.5 [t (C-13)], 28.9 [m, 3C (C-14, C-15, C16)], 30.9 [t (C-12)], 31.6 [t, C-17)], 68.0 [t (C-2)], 111.9 [d (C-8)], 116.7 [d (C-6)], 122.5 [d (C-5)], 124.6 [d (C-7)], 127.7 [s (C-10)], 143.4 [s (C-9)], 159.0 [s (C-3)], 169.0 [s (C-11)]. EIMS (probe, $70 \mathrm{eV}) \mathrm{m} / \mathrm{z} 305.4[\mathrm{M}]^{+}(29 \%)$.

4-Decanoyloxy-(2H)-1,4-benzoxazin-3(4H)-one (Dec-D-DIBOA): FTIR (cm $\left.{ }^{-\mathbf{1}}\right)$ : 2955 , 2926, 2855, 1708, 1497, 1277, 1052, 750; ${ }^{\mathbf{H}} \mathbf{N M R}\left(\mathbf{C D C l}_{\mathbf{3}}, \mathbf{4 0 0} \mathbf{M H z}\right) \delta 0.86[\mathrm{t}, 3 \mathrm{H}, \mathrm{J}$ $=7(\mathrm{H}-20)], 1.26[\mathrm{~m}, 10 \mathrm{H},(\mathrm{H}-19, \mathrm{H}-18, \mathrm{H}-17, \mathrm{H}-16, \mathrm{H}-15)], 1.41$ [dddd, 2H, J = 7, 15, 7, 15 (H-14)], 1.77 [dddd, 2H, J = 7, 15, 7, 15 (H-13)], 2.62 [dd, 2H, J = 7, 15 (H-12)], 4.74 [d, 2H, J = $13(\mathrm{H}-2)$ ], 6.82 [ddd, 1H, J = 8, 8, 2 (H-6)], 6.99 [m, 3H (H-5, H-7, H8)]. ${ }^{13} \mathbf{C ~ N M R ~}\left(\mathbf{C D C l}_{3}, \mathbf{1 0 0} \mathbf{M H z}\right) \delta 14.0$ [q (C-20)], 22.6 [t (C-19)], 24.6 [t (C-13)], 29.1 [m, 4C (C-17, C-16, C-15, C-14)], 31.1 [t (C-12)], 31.7 [t (C-18)], 68.1 [t (C-2)], 112.1 [d (C-8)], 116.9 [d (C-6)], 122.7 [d (C-5)], 124.8 [d (C-7)], 127.9 [s (C-10)], 143.6 [s (C-9)], 159.1 [s (C-3)], 169.2 [s (C-11)]. EIMS (probe, $70 \mathrm{eV}) \mathrm{m} / \mathrm{z} 319.3$ [M] $(5 \%)$. 
4-Undecanoyloxy-(2H)-1,4-benzoxazin-3(4H)-one (Undec-D-DIBOA): FTIR (cm $\left.{ }^{-\mathbf{1}}\right)$ : 2955, 2926, 2855, 1709, 1467, 1246, 1052, 752; ${ }^{\mathbf{1}} \mathbf{H} \mathbf{~ N M R}\left(\mathbf{C D C l}_{\mathbf{3}}, \mathbf{4 0 0} \mathbf{M H z}\right) \delta 0.86[\mathrm{t}$, $3 \mathrm{H}, \mathrm{J}=7$ (H-21)], 1.25 [m, 14H (H-20, H-19, H-18, H-17, H-16, H-15, H-14)], 1.77 [dddd, 2H, J = 7, 15, 7, 15 (H-13)], 2.63 [dd, 2H, J = 7, $15(\mathrm{H}-12)], 4.76$ [d, 2H, J = 9 (H-2)], 6.82 [ddd, 1H, J = 8, 8, 2 (H-6)], 6.98 [m, 3H (H-5, H-7, H-8)]. ${ }^{13} \mathbf{C}$ NMR $\left(\mathbf{C D C l}_{3}, 100 \mathrm{MHz}\right) \delta 14.1$ [q (C-21)], 22.6 [t (C-20)], 23.8 [t (C-13)], $29.2[\mathrm{~m}, 5 \mathrm{C}(\mathrm{C}-$ 18, C-17, C-16, C-15, C-14)], 31.1 [t (C-12)], 31.8 [t (C-19)], 68.1 [t (C-2)], 112.1 [d (C-8)], 116.8 [d (C-6)], 122.7 [d (C-5)], 124.8 [d (C-7)], 127.7 [s (C-10)], 143.5 [s (C9)], 159.2 [s (C-3)], 169.1 [s (C-11)]. EIMS (probe, $70 \mathrm{eV}) \mathrm{m} / z 333.3[\mathrm{M}]^{+}(5 \%)$.

4-Lauroyloxy-(2H)-1,4-benzoxazin-3(4H)-one (Lau-D-DIBOA): FTIR (cm $\left.{ }^{-1}\right)$ : 2955 , 2925, 2854, 1807, 1712, 1496, 1276, 1049, 748; ${ }^{\mathbf{1}} \mathbf{H} \mathbf{N M R}\left(\mathbf{C D C l}_{\mathbf{3}}, \mathbf{4 0 0} \mathbf{M H z}\right) \delta 0.86[\mathrm{t}$, $3 \mathrm{H}, \mathrm{J}=8(\mathrm{H}-22)], 1.24$ [m, 12H (H-21, H-20, H-19, H-18, H-17, H-16)], 1.42 [dddd, $2 \mathrm{H}, \mathrm{J}=7,15,7,15$ (H-15)], 1.60 [dddd, 2H, J = 7, 15, 7, 15 (H-14)], 1.78 [dddd, 2H, J $=7,15,7,15(\mathrm{H}-13)], 2.63[\mathrm{dd}, 2 \mathrm{H}, \mathrm{J}=7,15(\mathrm{H}-12)], 4.75$ [d, 2H, J = $13(\mathrm{H}-2)], 6.82$ $[\mathrm{ddd}, 1 \mathrm{H}, \mathrm{J}=8,8,2(\mathrm{H}-6)], 6.99[\mathrm{~m}, 3 \mathrm{H}(\mathrm{H}-5, \mathrm{H}-7, \mathrm{H}-8)] .{ }^{13} \mathbf{C} \mathbf{N M R}\left(\mathbf{C D C l}_{3}, \mathbf{1 0 0}\right.$ MHz) $\delta 14.0$ [q (C-22)], 22.6 [t (C-21)], 24.6 [t (C-13)], 29.3 [m, 5C (C-18, C-17, C-16, C-15, C-14)], 31.1 [t (C-19)], 31.8 [t (C-13)], 33.9 [t (C-20)], 68.1 [t (C-2)], 112.1 [d (C-8)], 116.9 [d (C-6)], 122.7 [d (C-5)], 124.7 [d (C-7)], 127.8 [s (C-10)], 143.6 [s (C9)], 159.2 [s (C-3)], 169.2 [s (C-11)]. EIMS (probe, $70 \mathrm{eV}$ ) m/z $347.4[\mathrm{M}]^{+}(47 \%)$.

4-Tridecanoyloxy-(2H)-1,4-benzoxazin-3(4H)-one (Tridec-D-DIBOA): FTIR ( $\left.\mathbf{c m}^{-1}\right)$ : 2935, 2803, 1801, 1730, 1492, 1270, 1049, 748; ${ }^{\mathbf{1}} \mathbf{H} \mathbf{~ N M R}\left(\mathbf{C D C l}_{3}, 400 \mathbf{M H z}\right) \delta 0.83$ [t, $3 \mathrm{H}, \mathrm{J}=7$ (H-23)], 1.22 [m, 18H (H-22, H-21, H-20, H-19, H-18, H-17, H-16, H-15, H14)], 1.74 [dddd, $2 \mathrm{H}, \mathrm{J}=7,15,7,15(\mathrm{H}-13)$ ], 2.61 [dd, 2H, J = 7, $15(\mathrm{H}-12)], 4.72$ [d, $2 \mathrm{H}, \mathrm{J}=8(\mathrm{H}-2)], 6.80$ [ddd, $1 \mathrm{H}, \mathrm{J}=8,8,2(\mathrm{H}-6)], 6.95[\mathrm{~m}, 3 \mathrm{H}(\mathrm{H}-5, \mathrm{H}-7, \mathrm{H}-8)] .{ }^{13} \mathbf{C}$ NMR $\left(\mathbf{C D C l}_{3}, \mathbf{1 0 0} \mathbf{M H z}\right) \delta 14.1$ [q (C-23)], 22.6 [t (C-22)], 24.6 [t (C-13)], 29.3 [m, 14C (C-20, C-19, C-18, C-17, C-16, C-15, C-14)], 31.0 [t (C-12)], 31.8 [t (C-21)], 68.0 [t (C-2)], 112.0 [d (C-8)], 116.8 [d (C-6)], 122.7 [d (C-5)], 124.7 [d (C-7)], 127.7 [s (C10)], 143.5 [s (C-9)], 159.1 [s (C-3)], 169.1 [s (C-11)]. EIMS (probe, $70 \mathrm{eV}$ ) m/z 361.3 $[\mathrm{M}]^{+}(5 \%)$.

4-Miristoyloxy-(2H)-1,4-benzoxazin-3(4H)-one (Mir-D-DIBOA): FTIR $\left(\mathbf{c m}^{-1}\right)$ : 2932 , 2834, 1815, 1703, 1499, 1268, 1053, 777; ${ }^{\mathbf{H}} \mathbf{H} \mathbf{N M R}\left(\mathbf{C D C l}_{3}, \mathbf{4 0 0} \mathbf{M H z}\right) \delta 0.86[\mathrm{t}, 3 \mathrm{H}, \mathrm{J}$ $=7(\mathrm{H}-24)], 1.26$ [m, 18H (H-23, H-22, H-21, H-20, H-19, H-18, H-17, H-16, H-15)], 1.41 [dddd, 2H, J = 7, 15, 7, 15 (H-14)], 1.77 [dddd, 2H, J = 7, 15, 7, 15 (H-13)], 2.62 [dd, 2H, J = 7, 15 (H-12)], 4.74 [d, 2H, J = 12 (H-2)], 6.81 [ddd, 1H, J = 8, 8, 2 (H-6)], 6.97 [m, 3H (H-5, H-7, H-8)]. ${ }^{13} \mathbf{C ~ N M R}\left(\mathbf{C D C l}_{3}, \mathbf{1 0 0} \mathbf{M H z}\right) \delta 14.0[\mathrm{q}(\mathrm{C}-24)], 22.6[\mathrm{t}$ (C-23)], 24.6 [t (C-14)], 29.2 [m, 8C (C-21, C-20, C-19, C-18, C-17, C-16, C-15, C13)], 31.0 [t (C-22)], 31.8 [t (C-12)], 68.1 [t (C-2)], 112.0 [d (C-8)], 116.8 [d (C-6)], 122.6 [d (C-5)], 124.7 [d (C-7)], 127.8 [s (C-10)], 143.5 [s (C-9)], 159.1 [s (C-3)], 169.1 [s (C-11)]. EIMS (probe, $70 \mathrm{eV}) \mathrm{m} / \mathrm{z} 375.5[\mathrm{M}]^{+}(37 \%)$.

Ethyl-2-[4'-methoxy-2'-nitrophenoxy]acetate: FTIR ( $\left.\mathbf{c m}^{-\mathbf{1}}\right)$ : 2983, 2942, 2844, 1755, $1609,1296,1060,839 .{ }^{1} \mathbf{H}$ NMR $\left(\mathbf{C D C l}_{3}, \mathbf{4 0 0} \mathbf{M H z}\right) \delta 1.28\left[\mathrm{t}, 3 \mathrm{H}, \mathrm{J}=7\left(\mathrm{CH}_{3} \mathrm{CH}_{2}-\right)\right]$, $3.81\left[\mathrm{~s}, 3 \mathrm{H}\left(\mathrm{CH}_{3} \mathrm{O}-\right)\right], 4.24\left[\mathrm{q}, 2 \mathrm{H}, \mathrm{J}=7\left(\mathrm{CH}_{3} \mathrm{CH}_{2^{-}}\right)\right], 4.72$ [s, $\left.2 \mathrm{H}(\mathrm{H}-2)\right], 7.04[\mathrm{~d}, 1 \mathrm{H}, \mathrm{J}$ 
$\left.=9\left(\mathrm{H}^{-} 6^{\prime}\right)\right], 7.09\left[\mathrm{dd}, 1 \mathrm{H}, \mathrm{J}=3,9\left(\mathrm{H}^{\prime} 5^{\prime}\right)\right], 7.36\left[\mathrm{~d}, 1 \mathrm{H}, \mathrm{J}=3\left(\mathrm{H}^{\prime} 3^{\prime}\right)\right] .{ }^{\mathbf{1 3}} \mathbf{C} \mathbf{N M R}\left(\mathbf{C D C l}_{3}\right.$, $100 \mathrm{MHz}) \delta 13.7\left[\mathrm{q}\left(\mathrm{CH}_{3} \mathrm{CH}_{2}-\right)\right.$ ], 55.7 [t $\left.\left(\mathrm{CH}_{3} \mathrm{O}-\right)\right], 61.2\left[\mathrm{t}\left(\mathrm{CH}_{3} \mathrm{CH}_{2}-\right)\right], 67.4[\mathrm{t}(\mathrm{C}-2)]$, 109.7 [d (C-3')], 117.7 [d (C-6')], 120.1 [d (C-5')], 140.4 [s (C-2')], 145.1 [s (C-1')], 153.7 [s (C-4')], 167.9 [s (C-1)]. EIMS (probe, $70 \mathrm{eV}) \mathrm{m} / \mathrm{z} 255.5[\mathrm{M}]^{+}(95 \%)$.

4-Hydroxy-6-methoxy-(2H)-1,4-benzoxazin-3(4H)-one (6-MeO-D-DIBOA): FTIR (cm$\left.{ }^{\mathbf{1}}\right)$ : 3090, 2896, 1694, 1651, 1506, 1210, 835, 804, 782. ${ }^{\mathbf{1}} \mathbf{H} \mathbf{~ N M R}\left(\mathbf{C D C l}_{\mathbf{3}}, \mathbf{4 0 0} \mathbf{~ M H z}\right)$ $\delta 3.70\left[\mathrm{~s}, 3 \mathrm{H}\left(\mathrm{CH}_{3} \mathrm{O}-\right)\right], 4.56[\mathrm{~s}, 2 \mathrm{H}(\mathrm{H}-2)], 6.44$ [dd, 1H, J = 3, $\left.9(\mathrm{H}-7)\right], 6.77$ [d, 1H, J $=9(\mathrm{H}-5)], 6.84[\mathrm{~d}, 1 \mathrm{H}, \mathrm{J}=3(\mathrm{H}-8)] .{ }^{13} \mathbf{C ~ N M R}\left(\mathbf{C D C l}_{3}, \mathbf{1 0 0} \mathbf{M H z}\right) \delta 55.8\left[\mathrm{q}\left(\mathrm{CH}_{3} \mathrm{O}-\right)\right]$, 68.1 [t (C-2)], 100.0 [d (C-5)], 109.0 [d (C-7)], 116.7 [d (C-8)], 129.6 [s (C-10)], 137.9 [s (C-9)], 155.5 [s (C-6)], 161.6 [s (C-3)]. EIMS (probe, $70 \mathrm{eV}) \mathrm{m} / \mathrm{z} 195.3$ [M] ${ }^{+}(100 \%)$.

4-Acetoxy-6-methoxy-(2H)-1,4-benzoxazin-3(4H)-one (6-MeO-ABOA): FTIR (cm $\left.{ }^{-1}\right)$ : 3249, 2929, 1692, 1514, 1198, 1051, 752. ${ }^{\mathbf{1}} \mathbf{H} \mathbf{~ N M R}\left(\mathbf{C D C l}_{3}, \mathbf{4 0 0} \mathbf{M H z}\right) \delta 2.38[\mathrm{~s}, 3 \mathrm{H}$, $(\mathrm{H}-12)], 3.75$ [s, 3H $\left.\left(\mathrm{CH}_{3} \mathrm{O}\right)\right], 4.71$ [s, 2H (H-2)], 6.54 [d, 1H, J = $\left.3(\mathrm{H}-5)\right], 6.61$ [dd, $1 \mathrm{H}, \mathrm{J}=3,8(\mathrm{H}-7)],, 6.93[\mathrm{~d}, 1 \mathrm{H}, \mathrm{J}=8(\mathrm{H}-8)] .{ }^{\mathbf{1 3}} \mathbf{C} \mathbf{N M R}\left(\mathbf{C D C l}_{3}, \mathbf{1 0 0} \mathbf{M H z}\right) \delta 17.5[\mathrm{q}$ $(\mathrm{C}-12)], 56.3$ [q $\left(\mathrm{CH}_{3} \mathrm{O}\right)$ ], 69.3 [t (C-2)], 100.4 [d (C-5)], 110.0 [d (C-7)], 118.3 [d (C8)], 131.9 [s (C-10)], 138.9 [s (C-9)], 155.4 [s (C-6)], 157.0 [s (C-3)], 168.0 [s (C-11)]. EIMS (probe, $70 \mathrm{eV}) \mathrm{m} / \mathrm{z} 237.1[\mathrm{M}]^{+}(21 \%)$.

4-Propanoyloxy-6-methoxy-(2H)-1,4-benzoxazin-3(4H)-one $\quad(P r-6-M e O-D-D I B O A):$ FTIR $\left(\mathbf{c m}^{-1}\right)$ : 3224, 2910, 1672, 1533, 1212, 1048, 749. ${ }^{\mathbf{1}} \mathbf{H} \mathbf{~ N M R}\left(\mathbf{C D C l}_{\mathbf{3}}, \mathbf{4 0 0} \mathbf{~ M H z}\right)$ $\delta 1.32[\mathrm{t}, 3 \mathrm{H}, \mathrm{J}=7(\mathrm{H}-13)], 2.69[\mathrm{q}, 2 \mathrm{H}, \mathrm{J}=7(\mathrm{H}-12)], 3.76\left[\mathrm{~s}, 3 \mathrm{H}\left(\mathrm{CH}_{3} \mathrm{O}\right)\right], 4.71[\mathrm{~d}$, $2 \mathrm{H}, \mathrm{J}=9(\mathrm{H}-2)], 6.40$ [d, 1H, J = 3 (H-5)], 6.52 [dd, 1H, J = 3, $9(\mathrm{H}-7)], 6.92[\mathrm{~d}, 1 \mathrm{H}, \mathrm{J}=$ $9(\mathrm{H}-8)] .{ }^{13} \mathbf{C}$ NMR $\left(\mathbf{C D C l}_{3}, \mathbf{1 0 0} \mathbf{M H z}\right) \delta 9.31[\mathrm{t}(\mathrm{C}-13)], 24.7[\mathrm{t}(\mathrm{C}-12)], 55.8[\mathrm{q}$ $\left(\mathrm{CH}_{3} \mathrm{O}\right)$ ], 68.3 [t $(\mathrm{C}-2)$ ], 99.5 [d (C-5)], 108.3 [d (C-7)], 117.3 [d (C-8)], 128.7 [s (C10)], 137.5 [s (C-9)], 155.4 [s (C-6)], 159.6 [s (C-3)], 166.4 [s (C-11)]. EIMS (probe, $70 \mathrm{eV}) \mathrm{m} / \mathrm{z} 251.6[\mathrm{M}]^{+}(2 \%)$.

4-Butanoyloxy-6-methoxy-(2H)-1,4-benzoxazin-3(4H)-one (Bu-6-MeO-D-DIBOA): FTIR $\left(\mathbf{c m}^{-1}\right): 3233,2958,1760,1690,1148,1042,750 .{ }^{1} \mathbf{H} \mathbf{~ N M R}\left(\mathbf{C D C l}_{3}, 400 \mathbf{~ M H z}\right)$ $\delta 1.05[\mathrm{t}, 3 \mathrm{H}, \mathrm{J}=7(\mathrm{H}-14)], 1.79$ [ddq, 2H, J = 6, 7, 14 (H-13)], 2.61 [dd, 2H, J = 6, 14 (H-12)], 3.72 [s, 3H ( $\left.\left.\mathrm{CH}_{3} \mathrm{O}\right)\right], 4.67$ [d, 2H, J = $\left.9(\mathrm{H}-2)\right], 6.39$ [d, 1H, J = $\left.3(\mathrm{H}-5)\right], 6.50$ $[\mathrm{dd}, 1 \mathrm{H}, \mathrm{J}=3,9(\mathrm{H}-7)], 6.89[\mathrm{~d}, 1 \mathrm{H}, \mathrm{J}=9(\mathrm{H}-8)] .{ }^{13} \mathbf{C} \mathbf{N M R}\left(\mathbf{C D C l}_{\mathbf{3}}, \mathbf{1 0 0} \mathbf{M H z}\right) \delta 13.5$ [q (C-14)], 18.3 [t (C-13)], 32.9 [t (C-12)], 55.6 [q $\left.\left(\mathrm{CH}_{3} \mathrm{O}\right)\right], 68.2$ [t (C-2)], 99.2 [d (C5)], 108.2 [d (C-7)], 117.2 [d (C-8)], 128.6 [s (C-10)], 137.4 [s (C-9)], 155.3 [s (C-6)], 159.6 [s (C-3)], 169.0 [s (C-11)]. EIMS (probe, $70 \mathrm{eV}) \mathrm{m} / \mathrm{z} 265.5[\mathrm{M}]^{+}(5 \%)$.

4-Valeroyloxy-6-methoxy-(2H)-1,4-benzoxazin-3(4H)-one (Bu-6-MeO-D-DIBOA): FTIR $\left(\mathbf{c m}^{-1}\right): 3248,2960,1765,1693,1153,1054,755 .{ }^{\mathbf{1}} \mathbf{H} \mathbf{~ N M R}\left(\mathbf{C D C l}_{3}, 400 \mathbf{~ M H z}\right)$ $\delta 0.96[\mathrm{t}, 3 \mathrm{H}, \mathrm{J}=7(\mathrm{H}-15)], 1.47$ [ddq, 2H, J = 6, 7, 14 (H-14)], 1.78 [dddd, 2H, J = 7, $14,7,14(\mathrm{H}-13)], 2.64[\mathrm{dd}, 2 \mathrm{H}, \mathrm{J}=7,14(\mathrm{H}-12)], 3.75\left[\mathrm{~s}, 3 \mathrm{H}\left(\mathrm{CH}_{3} \mathrm{O}\right)\right], 4.71[\mathrm{~d}, 2 \mathrm{H}, \mathrm{J}=$ $14(\mathrm{H}-2)], 6.40[\mathrm{~d}, 1 \mathrm{H}, \mathrm{J}=3(\mathrm{H}-5)], 6.52[\mathrm{dd}, 1 \mathrm{H}, \mathrm{J}=3,9(\mathrm{H}-7)], 6.91[\mathrm{~d}, 1 \mathrm{H}, \mathrm{J}=9(\mathrm{H}-$ 8)]. ${ }^{13} \mathrm{C}$ NMR $\left(\mathrm{CDCl}_{3}, \mathbf{1 0 0} \mathrm{MHz}\right) \delta 13.6$ [q (C-15)], 22.1 [t (C-14)], 26.7 [t (C-13)], 30.9 [t (C-12)], 55.8 [q $\left(\mathrm{CH}_{3} \mathrm{O}\right)$ ], 68.3 [t (C-2)], 99.4 [d (C-5)], 108.4 [d (C-7)], 117.3 [d (C-8)], 128.7 [s (C-10)], 137.5 [s (C-9)], 155.4 [s (C-6)], 159.8 [s (C-3)], 169.2 [s (C11)]. EIMS (probe, $70 \mathrm{eV}) \mathrm{m} / z 279.1[\mathrm{M}]^{+}(50 \%)$. 
4-Hexanoyloxy-6-methoxy-(2H)-1,4-benzoxazin-3(4H)-one (Bu-6-MeO-D-DIBOA): FTIR $\left(\mathbf{c m}^{-1}\right): 3235,2957,2932,1765,1702,1519,1153,1055,889.842,752 .{ }^{1} \mathbf{H}$ NMR $\left(\mathbf{C D C l}_{3}, 400 \mathrm{MHz}\right) \delta 0.88[\mathrm{t}, 3 \mathrm{H}, \mathrm{J}=7(\mathrm{H}-16)], 1.35$ [m, 4H (H-15, H-14)], 1.75 [dddd, $2 \mathrm{H}, \mathrm{J}=7,14,7,14(\mathrm{H}-13)], 2.60$ [dd, 2H, J = 7, $14(\mathrm{H}-12)], 3.70$ [s, 3H $\left.\left(\mathrm{CH}_{3} \mathrm{O}\right)\right], 4.65$ $[\mathrm{d}, 2 \mathrm{H}, \mathrm{J}=8(\mathrm{H}-2)], 6.36$ [d, 1H, J = $3(\mathrm{H}-5)], 6.47$ [dd, 1H, J = 3, $9(\mathrm{H}-7)], 6.87$ [d, 1H, $\mathrm{J}=9(\mathrm{H}-8)] \cdot{ }^{13} \mathbf{C}$ NMR $\left(\mathbf{C D C l}_{3}, \mathbf{1 0 0} \mathbf{M H z}\right) \delta 14.1[\mathrm{q}(\mathrm{C}-16)], 22.1[\mathrm{t}(\mathrm{C}-15)], 24.3[\mathrm{t}$ (C-13)], 31.0 [m, 2C (C-14, C-12)], 55.5 [q $\left(\mathrm{CH}_{3} \mathrm{O}\right)$ ], 68.1 [t (C-2)], 99.2 [d (C-5)], 108.2 [d (C-7)], 117.1 [d (C-8)], 128.5 [s (C-10)], 137.3 [s (C-9)], 155.2 [s (C-6)], 159.5 [s (C-3)], 168.9 [s (C-11)]. EIMS (probe, $70 \mathrm{eV}) \mathrm{m} / \mathrm{z} 293.1[\mathrm{M}]^{+}(41 \%)$.

4-Heptanoyloxy-6-methoxy-(2H)-1,4-benzoxazin-3(4H)-one (Hept-6-MeO-D-DIBOA): FTIR $\left(\mathbf{c m}^{-\mathbf{1}}\right):$ 2957, 2930, 2859, 1710, 1236, 810, 726. ${ }^{\mathbf{1}} \mathbf{H} \mathbf{N M R}\left(\mathbf{C D C l}_{\mathbf{3}}, \mathbf{4 0 0} \mathbf{M H z}\right)$ $\delta 0.88$ [t, 3H, J $=7(\mathrm{H}-17)], 1.31$ [m, 4H (H-16, H-15)], 1.42 [dddd, 2H, J = 7,13, 2, 14 (H-14)], 1.77 [dddd, 2H, J = 7, 15, 7, 15 (H-13)], 2.62 [dd, 2H, J = 7, 15 (H-12)], 3.73 $\left[\mathrm{s}, 3 \mathrm{H}\left(\mathrm{CH}_{3} \mathrm{O}\right)\right], 4.68$ [d, 2H, J = $\left.13(\mathrm{H}-2)\right], 6.39[\mathrm{~d}, 1 \mathrm{H}, \mathrm{J}=3(\mathrm{H}-5)], 6.50[\mathrm{dd}, 1 \mathrm{H}, \mathrm{J}=$ 3, 8 (H-7)], 6.89 [d, 1H, J = $8(\mathrm{H}-8)] .{ }^{13} \mathbf{C ~ N M R}\left(\mathbf{C D C l}_{\mathbf{3}}, \mathbf{1 0 0} \mathbf{M H z}\right) \delta 13.8$ [q (C-17)], 22.3 [t (C-16)], $24.6(\mathrm{C}-13)$ ], 28.6 [t (C-14)], 31.1 [t (C-15)], 31.2 [t (C-12)], 55.7 [q $\left(\mathrm{CH}_{3} \mathrm{O}\right)$ ], 68.2 [t $(\mathrm{C}-2)$ ], 99.3 [d (C-5)], 108.3 [d (C-7)], 117.2 [d (C-8)], 128.7 [s (C10)], 137.5 [s (C-9)], 155.3 [s (C-6)], 159.7 [s (C-3)], 169.1 [s (C-11)]. EIMS (probe, $70 \mathrm{eV}) \mathrm{m} / z 307.1[\mathrm{M}]^{+}(6 \%)$.

4-Octanoyloxy-6-methoxy-(2H)-1,4-benzoxazin-3(4H)-one (Oct-6-MeO-D-DIBOA): FTIR $\left(\mathbf{c m}^{-1}\right):$ 2933, 2850, 1712, 1502, 1277, 1054, 849. ${ }^{\mathbf{1}} \mathbf{H} \mathbf{~ N M R}\left(\mathbf{C D C l}_{\mathbf{3}}, \mathbf{4 0 0} \mathbf{~ M H z}\right)$ $\delta 0.85[\mathrm{t}, 3 \mathrm{H}, \mathrm{J}=7(\mathrm{H}-18)], 1.27$ [m, 6H (H-17, H-16, H-15)], 1.60 [dddd, 2H, J = 7, 15, 7, 15 (H-14)], 1.77 [dddd, 2H, J = 7, 15, 5, 14 (H-13)], 2.62 [d, 2H, J = 5 (H-12)], 3.73 [s, 3H $\left.\left(\mathrm{CH}_{3} \mathrm{O}\right)\right], 4.68$ [d, 2H, J = $\left.8(\mathrm{H}-2)\right], 6.39[\mathrm{~d}, 1 \mathrm{H}, \mathrm{J}=3(\mathrm{H}-5)], 6.50$ [dd, $1 \mathrm{H}, \mathrm{J}=3$, $9(\mathrm{H}-7)], 6.89[\mathrm{~d}, 1 \mathrm{H}, \mathrm{J}=9(\mathrm{H}-8)] .{ }^{13} \mathbf{C} \mathbf{N M R}\left(\mathbf{C D C l}_{\mathbf{3}}, \mathbf{1 0 0} \mathbf{M H z}\right) \delta 13.9$ [q (C-18)], 22.5 [t (C-17)], 24.6 [t (C-13)], 28.8 [m, 2C (C-15, C-14)], 31.5 [t (C-12)], 34.0 [t (C16)], 55.7 [q $\left(\mathrm{CH}_{3} \mathrm{O}\right)$ ], 68.2 [t (C-2)], 99.4 [d (C-5)], 108.4 [d (C-7)], 117.3 [d (C-8)], 128.6 [s (C-10)], 137.5 [s (C-9)], 155.3 [s (C-6)], 159.8 [s (C-3)], 169.1 [s (C-11)]. EIMS (probe, $70 \mathrm{eV}) \mathrm{m} / z 321.2[\mathrm{M}]^{+}(8 \%)$.

4-Nonanoyloxy-6-methoxy-(2H)-1,4-benzoxazin-3(4H)-one (Non-6-MeO-D-DIBOA): FTIR ( $\left.\mathbf{c m}^{-1}\right): 2952,2854,1710,1505,1266,1051,844 .{ }^{\mathbf{1}} \mathbf{H} \mathbf{~ N M R}\left(\mathbf{C D C l}_{3}, \mathbf{4 0 0} \mathbf{~ M H z}\right)$ $\delta 0.87$ [q, 3H, J = 6 (H- 19)], 1.26 [m, 8H (H-18, H-17, H-16, H-15)], 1.61 [dddd, 2H, J $=7,14,7,14(\mathrm{H}-14)], 1.78$ [dddd, $2 \mathrm{H}, \mathrm{J}=14,8,15,7(\mathrm{H}-13)], 2.63[\mathrm{dd}, 2 \mathrm{H}, \mathrm{J}=7,14$ $(\mathrm{H}-12)], 3.73$ [s, 3H $\left.\left(\mathrm{CH}_{3} \mathrm{O}\right)\right], 4.69$ [d, 2H, J = $\left.8(\mathrm{H}-2)\right], 6.40$ [d, 1H, J = $\left.2(\mathrm{H}-5)\right], 6.51$ $[\mathrm{dd}, 1 \mathrm{H}, \mathrm{J}=2,9(\mathrm{H}-6)], 6.90[\mathrm{~d}, 1 \mathrm{H}, \mathrm{J}=9(\mathrm{H}-8)] .{ }^{\mathbf{1 3}} \mathbf{C}$ NMR $\left(\mathbf{C D C l}_{3}, \mathbf{1 0 0} \mathbf{M H z}\right) \delta 14.1$ [q (C-19)], 22.7 [t (C-18)], 24.8 [t (C-13)], 29.1 [m, 3C (C-16 C-15, C-14], 31.2 [t (C17], 31.8 [t (C-12)], 55.8 [q $\left(\mathrm{CH}_{3} \mathrm{O}\right)$ ], 68.3 [t (C-2)], 99.4 [d (C-5)], 108.4 [d (C-7)], 117.3 [d (C-8)], 128.7[s (C-10)], 137.4 [s (C-9)], 155.3 [s (C-6)], 159.7 [s (C-3)], 169.1 [s (C-11)]. EIMS (probe, $70 \mathrm{eV}) \mathrm{m} / \mathrm{z} 179.1\left[\mathrm{M}-\mathrm{CH}_{3}\left(\mathrm{CH}_{2}\right)_{7} \mathrm{CO}_{2}\right]^{+}(100 \%)$.

4-Decanoyloxy-6-methoxy-(2H)-1,4-benzoxazin-3(4H)-one (Dec-6-MeO-D-DIBOA): FTIR $\left(\mathbf{c m}^{-1}\right): 2956,2855,1721,1505,1267,1051.832 .{ }^{1} \mathbf{H} \mathbf{~ N M R}\left(\mathbf{C D C l}_{\mathbf{3}}, \mathbf{4 0 0} \mathbf{~ M H z}\right)$ $\delta 0.85$ [q, 3H, J $=7$ (H-20)], 1.24 [m, 10H (H-19, H-18, H-17, H-16, H-15)], 1.69 [dddd, 2H, J = 7, 15, 7, 14 (H-14)], 1.77 [dddd, 2H, J = 7, 15, 7, 14 (H-13)], 2.62 [dd, $2 \mathrm{H}, \mathrm{J}=7,14(\mathrm{H}-12)], 3.72\left[\mathrm{~s}, 3 \mathrm{H}\left(\mathrm{CH}_{3} \mathrm{O}\right)\right], 4.69$ [d, 2H, J = $\left.8(\mathrm{H}-2)\right], 6.39$ [d, 1H, J = 3 $(\mathrm{H}-5)], 6.50[\mathrm{dd}, 1 \mathrm{H}, \mathrm{J}=3,9(\mathrm{H}-6)], 6.89[\mathrm{~d}, 1 \mathrm{H}, \mathrm{J}=9(\mathrm{H}-8)] .{ }^{\mathbf{1 3}} \mathbf{C} \mathbf{N M R}\left(\mathbf{C D C l}_{3}, \mathbf{1 0 0}\right.$ MHz) $\delta 14.0$ [q (C-20)], 22.6 [t (C-19)], 24.6 [t [C-13)], 29.1 [m, 4C (C-17, C-16, C-15, 
C-14)], 31.1 [t (C-12)], 31.8 [t (C-18)], 55.6 [q $\left(\mathrm{CH}_{3} \mathrm{O}\right)$ ], 68.2 [t (C-2)], 99.3 [d (C-5)], 108.3 [d (C-7)], 117.2 [d (C-8)], 128.6 [s (C-10)], 137.5 [s (C-9)], 155.3 [s (C-6)], 159.8 [s (C-3)], 169.1 [s (C-11)]. EIMS (probe, $70 \mathrm{eV}) \mathrm{m} / z$ 179.1 $\left[\mathrm{M}-\mathrm{CH}_{3}\left(\mathrm{CH}_{2}\right)_{8} \mathrm{CO}_{2}\right]^{+}$ $(30 \%) ; 155.1\left[\mathrm{CH}_{3}\left(\mathrm{CH}_{2}\right)_{8} \mathrm{CO}_{2}\right]^{+}(9 \%)$.

4-Undecanoyloxy-6-methoxy-(2H)-1,4-benzoxazin-3(4H)-one (Undec-6-MeO-DDIBOA): FTIR $\left(\mathbf{c m}^{-1}\right): 2947,2850,1722,1519,1275,1054.830 .{ }^{1} \mathbf{H}$ NMR $\left(\mathbf{C D C l}_{3}\right.$, $400 \mathrm{MHz}) \delta 0.85$ [q, 3H, J = 7 (H-21)], 1.23 [m, 12H (H-20, H-19, H-18, H-17, H-16, $\mathrm{H}-15)$ ], 1.59 [dddd, 2H, J = 7, 15, 7, 14 (H-14)], 1.76 [dddd, 2H, J = 7, 15, 7, 14 (H13)], $2.61[\mathrm{dd}, 2 \mathrm{H}, \mathrm{J}=7,14(\mathrm{H}-12)], 3.72$ [s, 3H $\left.\left(\mathrm{CH}_{3} \mathrm{O}\right)\right], 4.69$ [d, 2H, J = $\left.8(\mathrm{H}-2)\right]$, $6.38[\mathrm{~d}, 1 \mathrm{H}, \mathrm{J}=3(\mathrm{H}-5)], 6.49[\mathrm{dd}, 1 \mathrm{H}, \mathrm{J}=3,9(\mathrm{H}-6)], 6.88[\mathrm{~d}, 1 \mathrm{H}, \mathrm{J}=9(\mathrm{H}-8)] .{ }^{13} \mathbf{C}$ NMR (CDCl 3 , 100 MHz) $\delta 14.0$ [q (C-21)], 22.6 [t (C-20)], 24.6 [t [C-13)], 29.1 [m, 5C (C-18, C-17, C-16, C-15, C-14)], 31.0 [t (C-12)], 31.8 [t (C-19)], 55.6 [q $\left(\mathrm{CH}_{3} \mathrm{O}\right)$ ], 68.2 [t (C-2)], 99.3 [d (C-5)], 108.3 [d (C-7)], 117.2 [d (C-8)], 128.6 [s (C-10)], 137.4 [s (C-9)], 155.3 [s (C-6)], 159.8 [s (C-3)], 169.1 [s (C-11)]. EIMS (probe, $70 \mathrm{eV}$ ) m/z $363.3[\mathrm{M}]^{+}(5 \%)$.

4-Lauroyloxy-6-methoxy-(2H)-1,4-benzoxazin-3(4H)-one (Lau-6-MeO-D-DIBOA): FTIR $\left(\mathbf{c m}^{-1}\right):$ 2956, 2848, 1720, 1532, 1270, 1050. 822. ${ }^{\mathbf{1}} \mathbf{H} \mathbf{~ N M R}\left(\mathbf{C D C l}_{3}, \mathbf{4 0 0} \mathbf{~ M H z}\right)$ $\delta 0.85[\mathrm{q}, 3 \mathrm{H}, \mathrm{J}=7(\mathrm{H}-22)], 1.24$ [m, 14H $(\mathrm{H}-21, \mathrm{H}-20, \mathrm{H}-19, \mathrm{H}-18, \mathrm{H}-17, \mathrm{H}-16, \mathrm{H}-$ 15)], 1.60 [dddd, 2H, J = 7, 15, 7, 14 (H-14)], 1.77 [dddd, 2H, J = 7, 15, 7, 14 (H-13)], $2.62[\mathrm{dd}, 2 \mathrm{H}, \mathrm{J}=7,14(\mathrm{H}-12)], 3.73\left[\mathrm{~s}, 3 \mathrm{H}\left(\mathrm{CH}_{3} \mathrm{O}\right)\right], 4.69$ [d, 2H, J = $\left.8(\mathrm{H}-2)\right], 6.39$ [d, $1 \mathrm{H}, \mathrm{J}=3(\mathrm{H}-5)], 6.50[\mathrm{dd}, 1 \mathrm{H}, \mathrm{J}=3,9(\mathrm{H}-6)], 6.89[\mathrm{~d}, 1 \mathrm{H}, \mathrm{J}=9(\mathrm{H}-8)] .{ }^{13} \mathbf{C} \mathbf{N M R}$ $\left(\mathbf{C D C l}_{3}, 100 \mathrm{MHz}\right) \delta 14.0$ [q (C-22)], 22.6 [t (C-21)], 24.6 [t [C-13)], 29.1 [m, 5C (C19, C-18, C-17, C-16, C-15, C-14)], 31.8 [t (C-12)], 34.0 [t (C-20)], 55.6 [q $\left.\left(\mathrm{CH}_{3} \mathrm{O}\right)\right]$, 68.2 [t (C-2)], 99.3 [d (C-5)], 108.3 [d (C-7)], 117.2 [d (C-8)], 128.6 [s (C-10)], 137.4 [s (C-9)], 155.3 [s (C-6)], 159.8 [s (C-3)], 169.1 [s (C-11)]. EIMS (probe, $70 \mathrm{eV}) \mathrm{m} / \mathrm{z}$ $377.2[\mathrm{M}]^{+}(2 \%)$.

4-Tridecanoyloxy-6-methoxy-(2H)-1,4-benzoxazin-3(4H)-one (Tridec-6-MeO-DDIBOA): FTIR $\left(\mathbf{c m}^{-1}\right): 2910,2844,1715,1508,1217,1054,726 .{ }^{1} \mathbf{H}$ NMR $\left(\mathbf{C D C l}_{3}\right.$, 400 MHz) $\delta 0.85$ [q, 3H, J = 7 (H-23)], 1.24 [m, 16H (H-22, H-21, H-20, H-19, H-18, H-17, H-16, H-15)], 1.59 [dddd, 2H, J = 7, 15, 7, 14 (H-14)], 1.77 [dddd, 2H, J = 7, 15, 7, $14(\mathrm{H}-13)], 2.62[\mathrm{dd}, 2 \mathrm{H}, \mathrm{J}=7,14(\mathrm{H}-12)], 3.73$ [s, 3H $\left.\left(\mathrm{CH}_{3} \mathrm{O}\right)\right], 4.69$ [d, 2H, J = 7 (H-2)], 6.39 [d, 1H, J = $3(\mathrm{H}-5)], 6.50$ [dd, 1H, J = 3, $9(\mathrm{H}-6)], 6.89$ [d, 1H, J = $9(\mathrm{H}-8)]$. ${ }^{13} \mathbf{C ~ N M R}\left(\mathrm{CDCl}_{3}, \mathbf{1 0 0} \mathbf{M H z}\right) \delta 14.0$ [q (C-23)], 22.6 [t (C-22)], 24.6 [t [C-13)], 29.1 [m, 6C (C-21, C-19, C-18, C-17, C-16, C-15, C-14)], 31.8 [t (C-12)], 34.0 [t (C-21)], 55.6 [q $\left(\mathrm{CH}_{3} \mathrm{O}\right)$ ], 68.2 [t (C-2)], 99.4 [d (C-5)], 108.3 [d (C-7)], 117.2 [d (C-8)], 128.6 [s (C-10)], 137.5 [s (C-9)], 155.3 [s (C-6)], 159.7 [s (C-3)], 169.1 [s (C-11)]. EIMS (probe, $70 \mathrm{eV}) \mathrm{m} / \mathrm{z} 391.3[\mathrm{M}]^{+}(5 \%)$.

4-Miristoyloxy-6-methoxy-(2H)-1,4-benzoxazin-3(4H)-one (Mir-6-MeO-D-DIBOA): FTIR $\left(\mathbf{c m}^{-1}\right)$ : 2917, 2849, 1700, 1505, 1207, 1051, 720. ${ }^{\mathbf{1}} \mathbf{H} \mathbf{~ N M R}\left(\mathbf{C D C l}_{\mathbf{3}}, \mathbf{4 0 0} \mathbf{~ M H z}\right)$ $\delta 0.86$ [q, 3H, J $=7$ (H-24)], 1.24 [m, 18H (H-23, H-22, H-21, H-20, H-19, H-18, H-17, H-16, H-15)], 1.60 [dddd, 2H, J = 7, 15, 7, 14 (H-14)], 1.78 [dddd, 2H, J = 7, 15, 7, 14 $(\mathrm{H}-13)], 2.62$ [dd, 2H, J = 7, $14(\mathrm{H}-12)], 3.74$ [s, 3H $\left.\left(\mathrm{CH}_{3} \mathrm{O}\right)\right], 4.69$ [d, 2H, J = $\left.7(\mathrm{H}-2)\right]$, $6.39[\mathrm{~d}, 1 \mathrm{H}, \mathrm{J}=3(\mathrm{H}-5)], 6.51[\mathrm{dd}, 1 \mathrm{H}, \mathrm{J}=3,9(\mathrm{H}-6)], 6.89[\mathrm{~d}, 1 \mathrm{H}, \mathrm{J}=9(\mathrm{H}-8)] .{ }^{13} \mathbf{C}$ NMR $\left(\mathbf{C D C l}_{3}, \mathbf{1 0 0} \mathrm{MHz}\right) \delta 14.0$ [q (C-24)], 22.6 [t (C-23)], 24.7 [t [C-14)], $29.1[\mathrm{~m}$, 7C (C-21, C-20, C-19, C-18, C-17, C-16, C-15)], 31.8 [t (C-12)], 34.0 [t (C-22)], 55.7 [q $\left.\left(\mathrm{CH}_{3} \mathrm{O}\right)\right], 68.3$ [t $\left.(\mathrm{C}-2)\right], 99.4$ [d (C-5)], 108.4 [d (C-7)], 117.3 [d (C-8)], 128.6 [s (C- 
10)], 137.5 [s (C-9)], 155.3 [s (C-6)], 159.7 [s (C-3)], 169.1 [s (C-11)]. EIMS (probe, $70 \mathrm{eV}) \mathrm{m} / \mathrm{z} 405.3[\mathrm{M}]^{+}$(3\%). 


\section{Phytotoxicity data}

Lepidium sativum L. (cress)
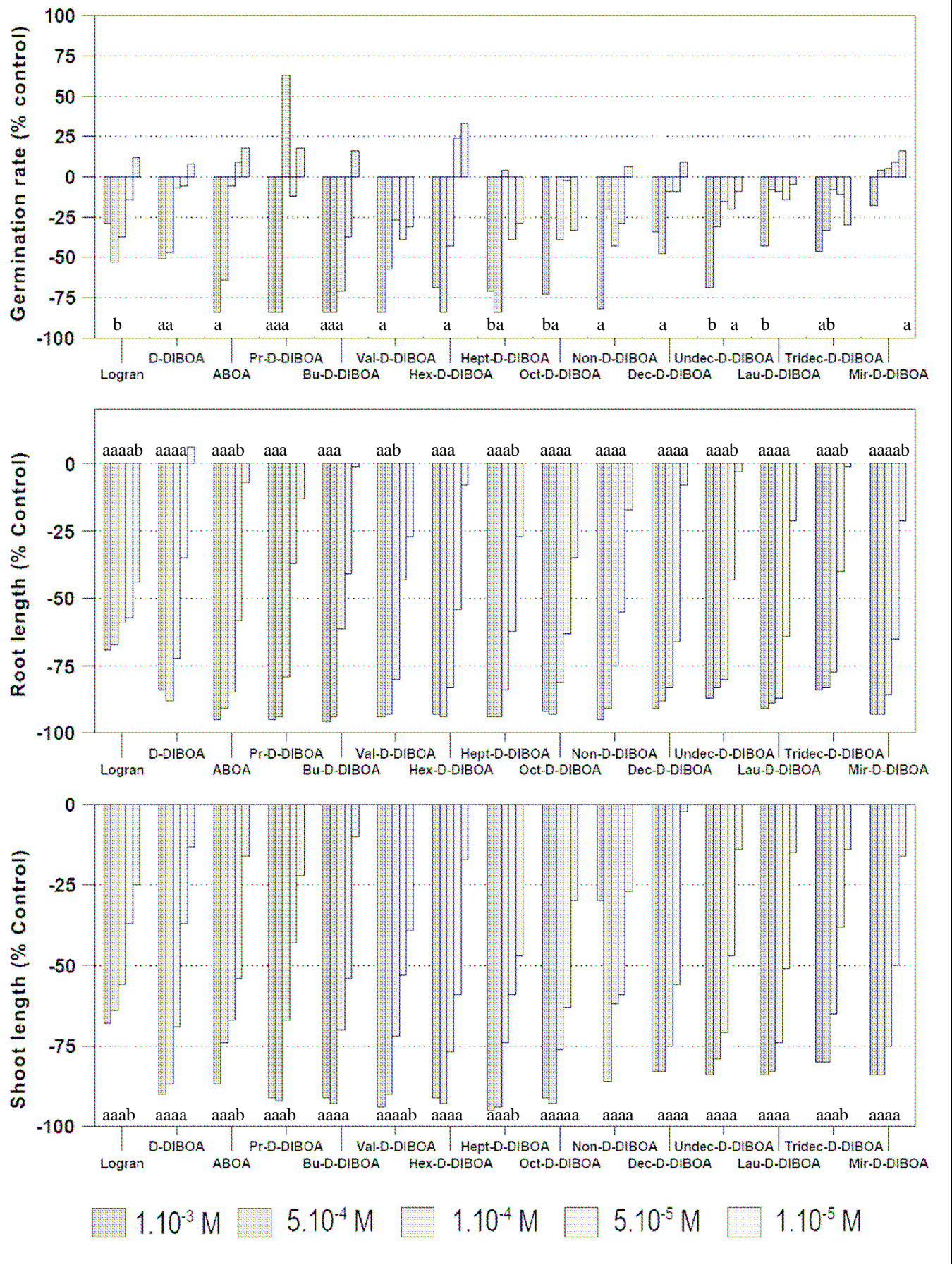


\section{Lactuca sativa L. (lettuce)}
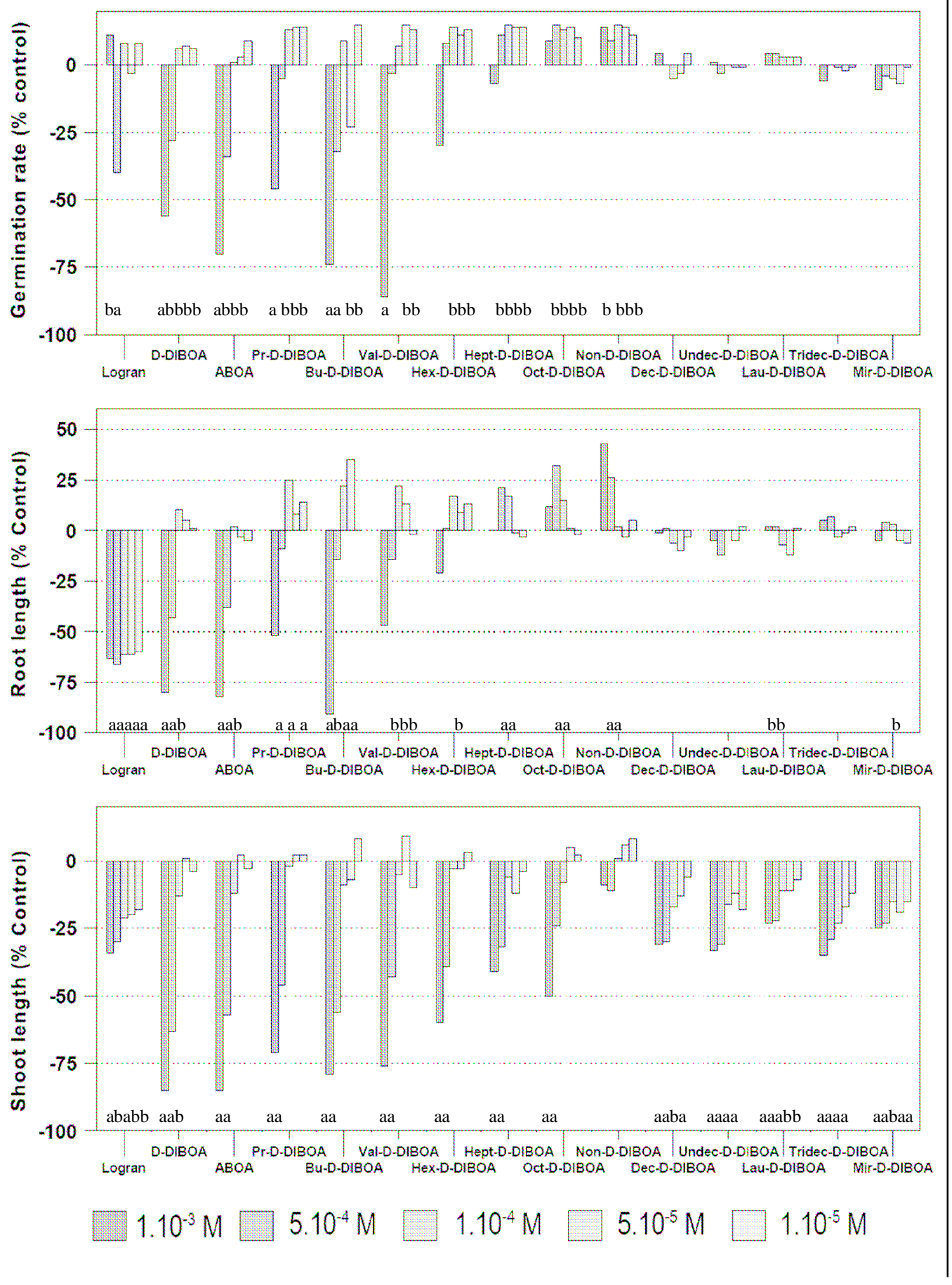
Lycopersicon esculentum Will. (tomato).
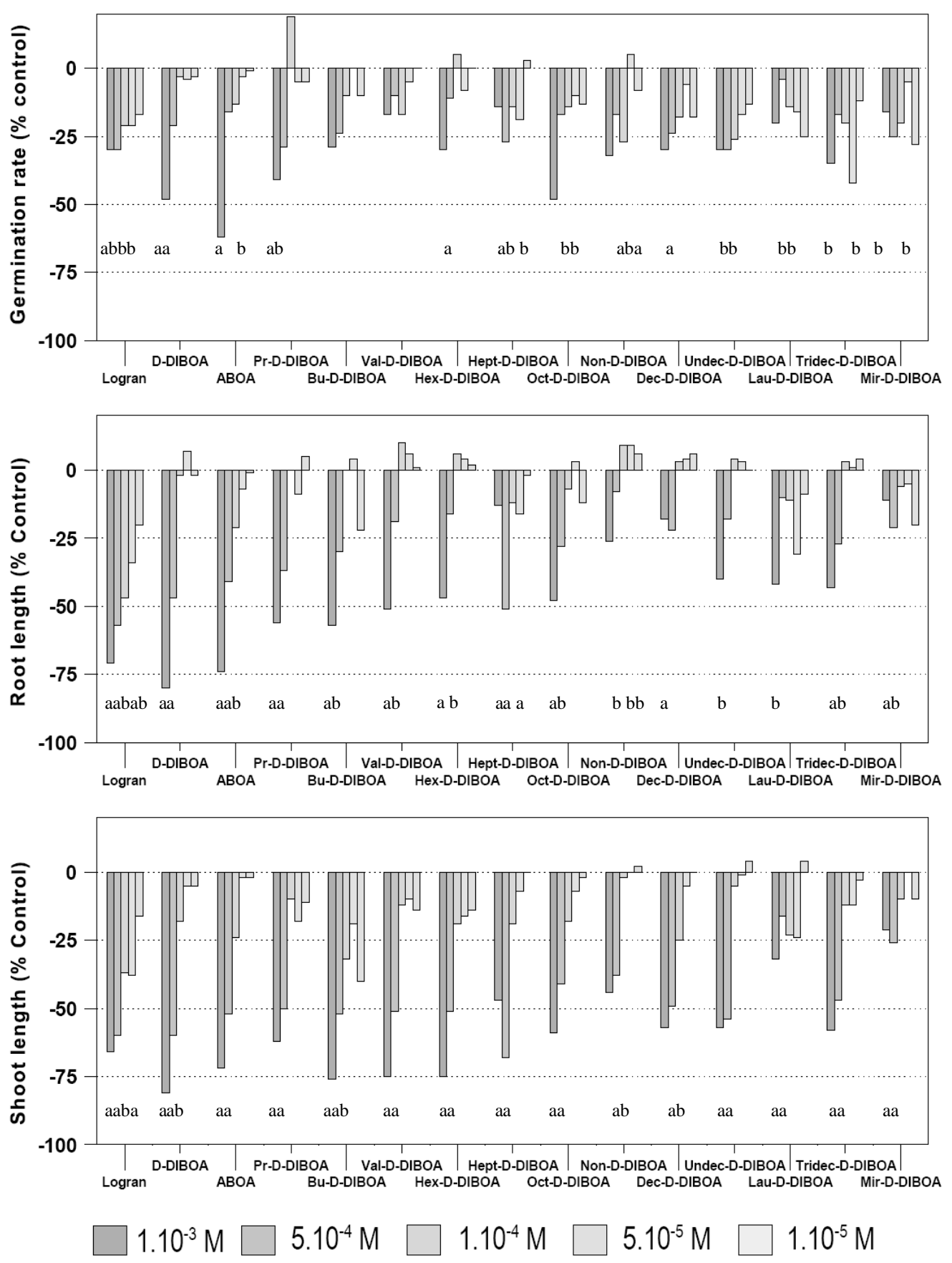
Allium cepa L. (onion)
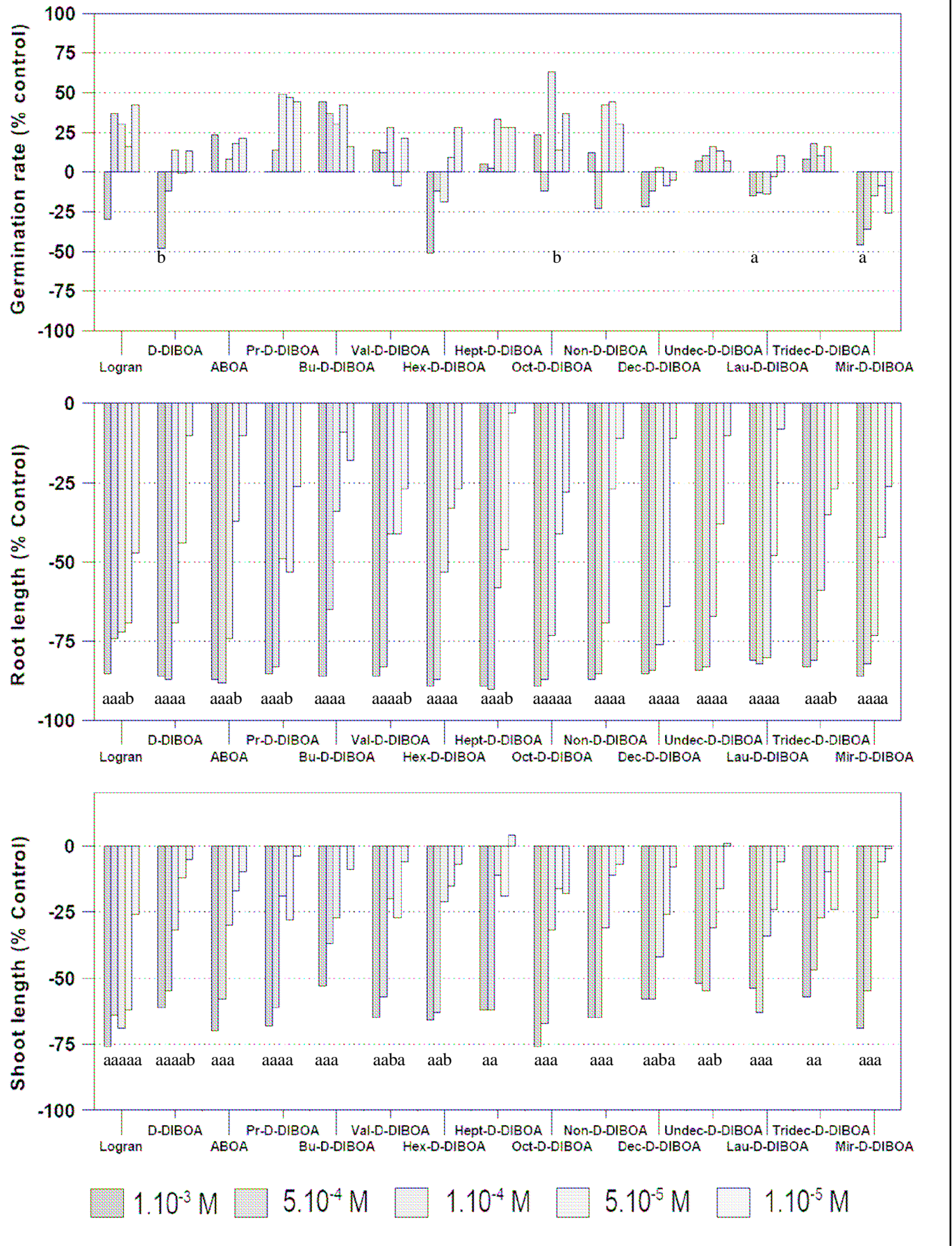
Triticum aestivum L. (wheat)
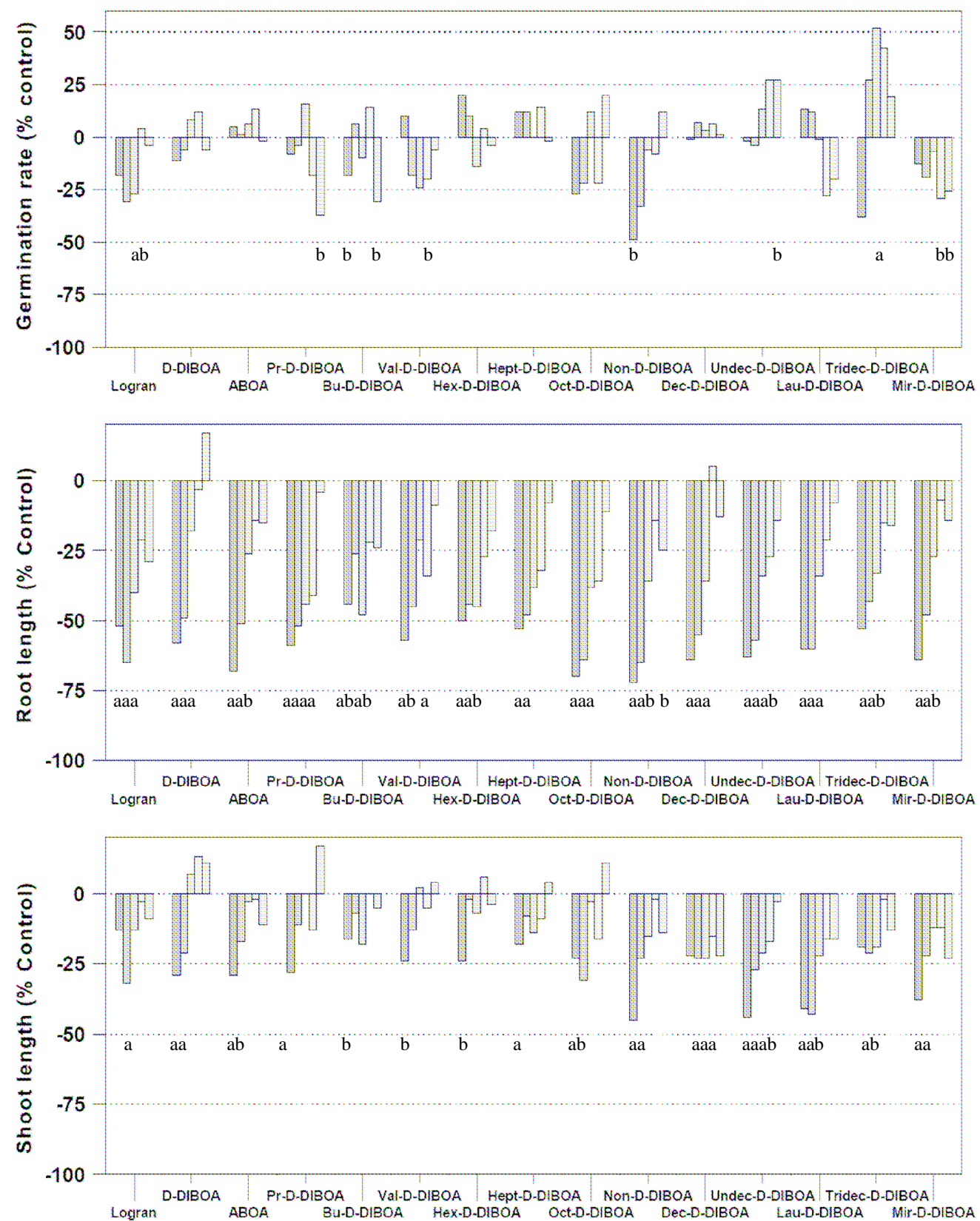
$1.10^{-3} \mathrm{M}$
$5.10^{-4} \mathrm{M}$
$1.10^{-4} \mathrm{M}$
$5.10^{-5} \mathrm{M}$
$1.10^{-5} \mathrm{M}$ 
Lolium rigidum Gaud. (rigid ryegrass)
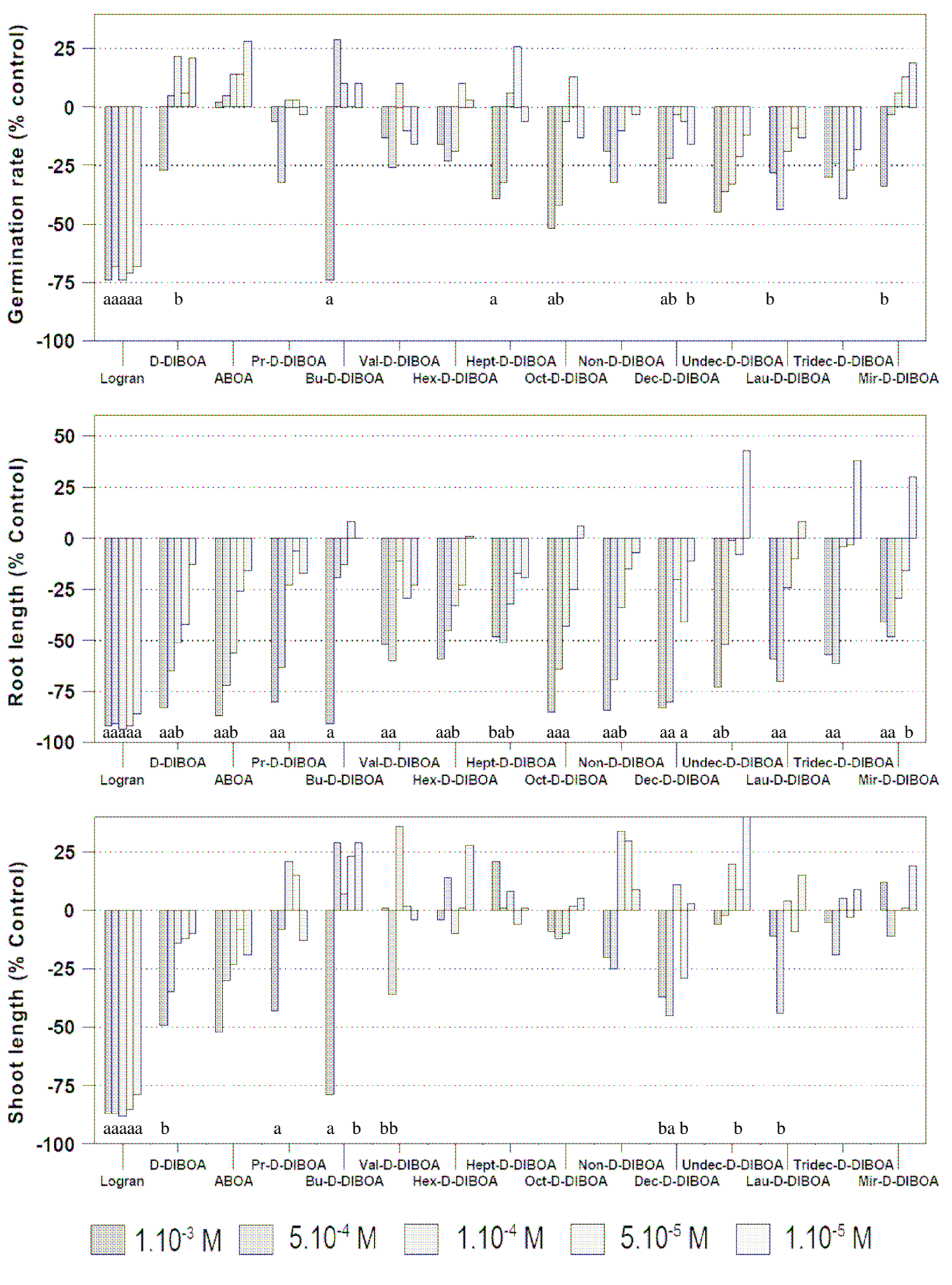
Avena fatua L. (wild oat).
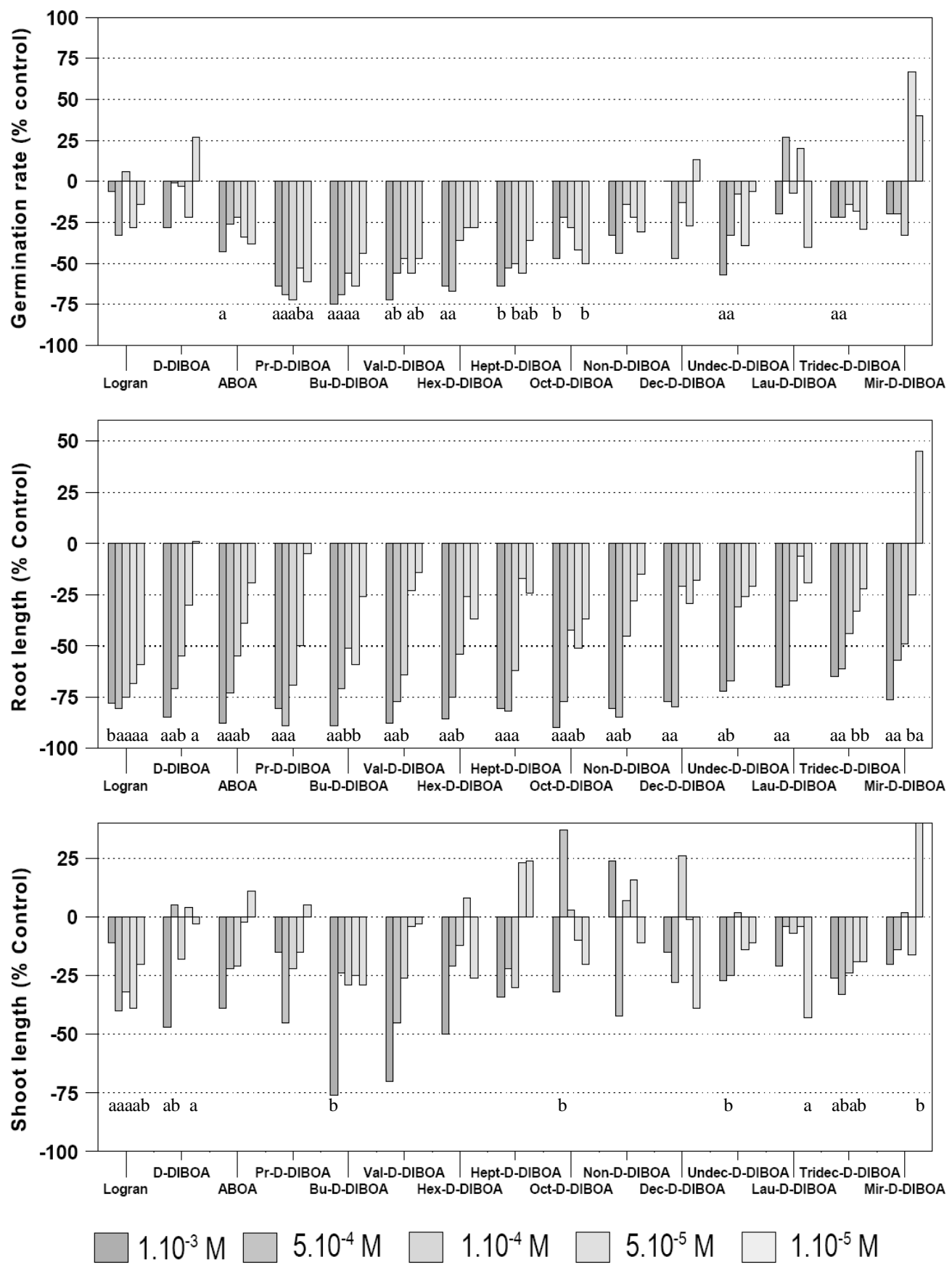
Avena fatua L. (wild oat, 6-methoxy-D-DIBOA esters)

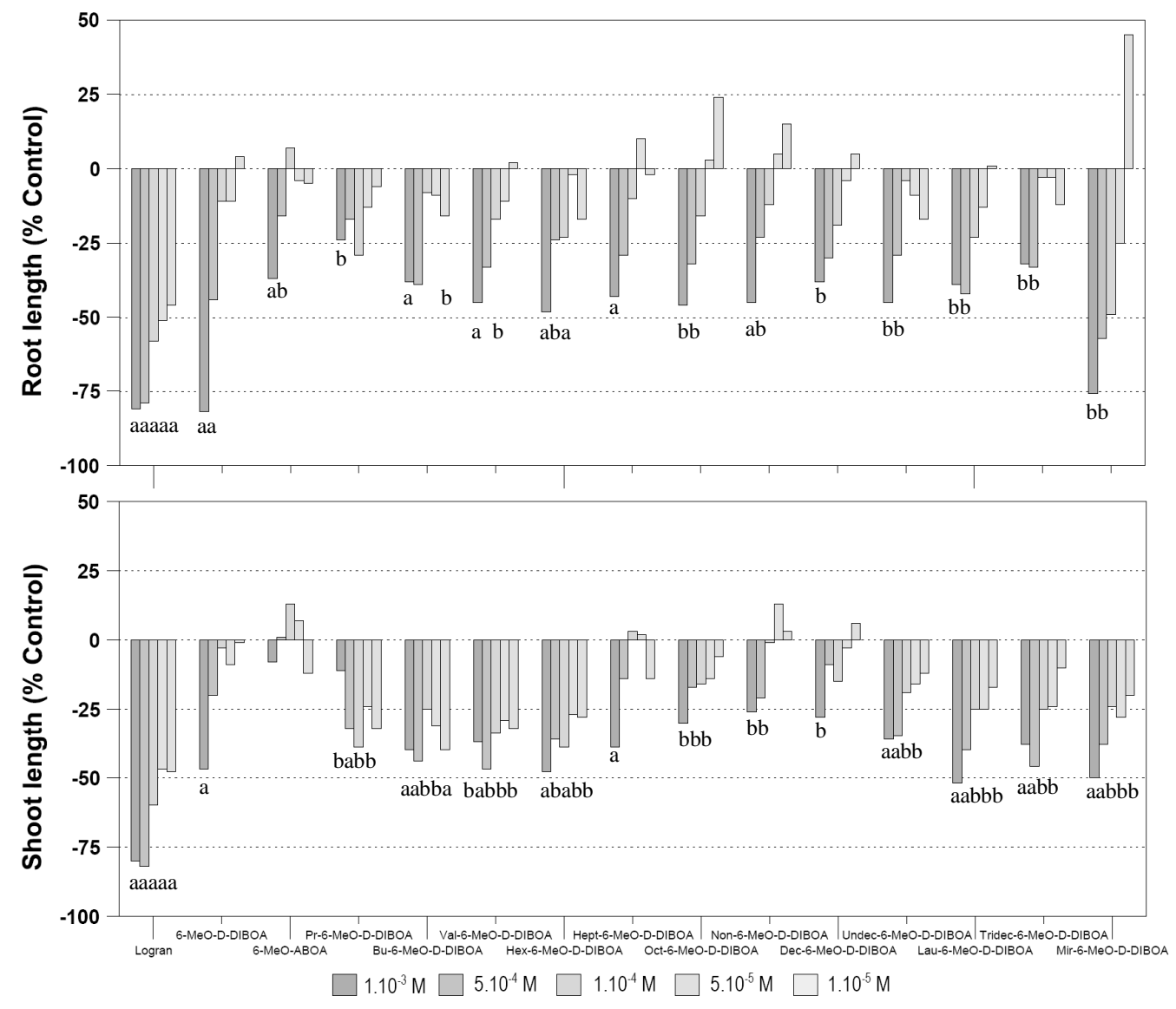

